\title{
A BEST CONSTANT FOR ZYGMUND'S CONJUGATE FUNCTION INEQUALITY
}

\author{
COLIN BENNETT 1
}

Abstract. When the space $L \log ^{+} L$ is given the Hardy-Littlewood norm the best constant in the corresponding version of Zygmund's conjugate function inequality is shown to be

$$
\mathbf{K}=\frac{1^{-2}-3^{-2}+5^{-2}-7^{-2}+\cdots}{1^{-2}+3^{-2}+5^{-2}+7^{-2}+\cdots} .
$$

This complements the recent result of Burgess Davis that the best constant in Kolmogorov's inequality is $\mathbf{K}^{-1}$.

The symbol $\mathbf{K}$ will be used throughout for the constant

$$
\mathbf{K}=\frac{1^{-2}-3^{-2}+5^{-2}-7^{-2}+\cdots}{1^{-2}+3^{-2}+5^{-2}+7^{-2}+\cdots}=0.7424537 \cdots .
$$

Let $f$ be a real-valued $2 \pi$-periodic function of class $L^{1}(0,2 \pi)=L^{1}$, and let $\tilde{f}$ be its conjugate function [10, Chapter IV]. It is well known that $\tilde{f}$ is finite a.e. on $(0,2 \pi)$ but need not itself be integrable. There is however the following weak-type estimate for $\tilde{f}$ due to Kolmogorov [10, p. 134], the best constant for which was determined recently by Davis [5] (cf. also [4]).

THeORem 1 (Kolmogorov, Davis). When $f \in L^{1}$ the conjugate function $\tilde{f}$ satisfies the inequality

$$
y m\{x:|\tilde{f}(x)|>y\} \leqslant \mathbf{K}^{-1} \int_{0}^{2 \pi}|f(x)| d x, \quad y>0,{ }^{2}
$$

and the constant $\mathbf{K}^{-1}$ is best-possible.

Complementing Kolmogorov's inequality is the result of Zygmund [10, p. 254] that the conjugate function $\tilde{f}$ is integrable whenever $f$ is of class $L \log ^{+} L$. The admissible constants in Zygmund's inequality have been determined by Pichorides [8] as follows.

TheOREM 2 (Zygmund, Pichorides). There are absolute constants $A$ and $B=B(A)$ such that for every $f \in L \log ^{+} L$ the conjugate function $\tilde{f}$ satisfies the inequality

$$
\int_{0}^{2 \pi}|\tilde{f}(x)| d x \leqslant A \int_{0}^{2 \pi}|f(x)| \log ^{+}|f(x)| d x+B,
$$

Received by the editors December 18, 1974 and, in revised form, March 20, 1975 and June 23, 1975.

AMS (MOS) subject classifications (1970). Primary 42A40.

1 Supported by NSF MPS 74/17845.

$2 m(E)$ denotes the Lebesgue measure of a measurable subset $E$ of $(0,2 \pi)$. 
and the admissible values for $A$ are precisely those that exceed $2 / \pi$.

In the following version of Zygmund's inequality, established by O'Neil and Weiss [7], the functional on the right of (2) is replaced by a norm for the space $L \log ^{+} L$.

TheOREM 3 (O'NeIL, Weiss). When $f \in L \log ^{+} L$ the conjugate function $\tilde{f}$ satisfies the inequality

$$
\int_{0}^{2 \pi}|\tilde{f}(x)| d x \leqslant \frac{2}{\pi} \int_{0}^{2 \pi} f^{*}(t) \log \cot (t / 8) d t,
$$

and the constant $2 / \pi$ is best-possible.

Here we have used $f^{*}$ to denote the decreasing rearrangement on $(0,2 \pi)$ of $f$. Observe, for instance by the argument used in [10, p. 33] or in [1, Proof of Theorem 4.1], that the functional on the right of (3) is finite if and only if $f \in L \log ^{+} L$, and that it defines a complete norm (a Lorentz $\Lambda$-norm) on $L \log ^{+} L$ because $\log \cot (t / 8)$ is decreasing on $(0,2 \pi)[6$, Theorem 1$]$.

An equivalent norm which is somewhat easier to manipulate (cf. [1], [2], [3]) is the Hardy-Littlewood norm

$$
f \rightarrow \int_{0}^{2 \pi} f^{*}(t) \log (2 \pi / t) d t=\int_{0}^{2 \pi} f^{* *}(t) d t, \quad f \in L \log ^{+} L,
$$

where $f^{* *}(t)=t^{-1} \int_{0}^{t} f^{*}(s) d s, 0<t<2 \pi$. Apart from a multiplicative constant this is, in the notation of [1], the norm of the Lorentz space $L^{11}$. Since $f^{* *}$ is the one-sided Hardy-Littlewood maximal function of $f^{*}$ the equality in (4), established by integrating by parts, is merely a reflection of the HardyLittlewood-Stein-Herz theorem that the maximal function is integrable precisely when $f \in L \log ^{+} L$ (cf. [1] for more details).

We come now to our main result. In contrast to Davis' proof of Theorem 1, which uses Brownian motion, ours is quite elementary although it does proceed from the O'Neil-Weiss inequality (3) and hence ultimately depends on the Stein-Weiss theory for conjugate functions [9].

THEOREM 4. When $f \in L \log ^{+} L$ the conjugate function satisfies the inequality

$$
\int_{0}^{2 \pi}|\tilde{f}(x)| d x \leqslant \mathbf{K} \int_{0}^{2 \pi} f^{* *}(t) d t,
$$

and the constant $\mathbf{K}$ is best-possible.

Proof. From the obvious inequality $\tan (t / 8)<t / 2 \pi$, if $0<t<2 \pi$, it follows that

$$
\alpha \equiv \frac{1}{2 \pi} \int_{0}^{2 \pi} \log \cot (t / 8) d t>\frac{1}{2 \pi} \int_{0}^{2 \pi} \log (2 \pi / t) d t=1 .
$$

We claim that the inequality

$$
\int_{0}^{s} \log \cot (t / 8) d t \leqslant \alpha \int_{0}^{s} \log (2 \pi / t) d t
$$

holds for every $s$ with $0 \leqslant s \leqslant 2 \pi$; equivalently, if we set 


$$
F(t)=\log \left\{(2 \pi / t)^{\alpha} \tan (t / 8)\right\}, \quad 0 \leqslant t \leqslant 2 \pi,
$$

then

$$
\int_{0}^{s} F(t) d t \geqslant 0, \quad 0 \leqslant s \leqslant 2 \pi .
$$

In fact, from (6) we see that $F(0+)=\infty$ and $F(2 \pi)=0$ so an examination of the derivative $F^{\prime}$ yields the existence of a number $s_{0} \in(0,2 \pi)$ such that $F$ is positive on $\left(0, s_{0}\right)$ and negative on $\left(s_{0}, 2 \pi\right)$. The validity of $(8)$ is therefore clear if $0 \leqslant s \leqslant s_{0}$. Furthermore, if $s_{0}<s<2 \pi$, then by (6) we have

$$
\int_{0}^{s} F(t) d t=\int_{0}^{2 \pi} F(t) d t-\int_{s}^{2 \pi} F(t) d t=-\int_{s}^{2 \pi} F(t) d t \geqslant 0 .
$$

This establishes the inequalities (7) and (8).

We next use a well-known device (involving nothing more than an integration by parts, cf. [6, Lemma 1]) whereby we can introduce into the integrands in (7) any nonnegative decreasing function without violating the inequality. In particular, for any $f \in L \log ^{+} L$,

$$
\int_{0}^{2 \pi} f^{*}(t) \log \cot (t / 8) d t \leqslant \alpha \int_{0}^{2 \pi} f^{*}(t) \log (2 \pi / t) d t=\alpha \int_{0}^{2 \pi} f^{* *}(t) d t .
$$

It now follows from (3) that

$$
\int_{0}^{2 \pi}|\tilde{f}(x)| d x \leqslant \frac{2 \alpha}{\pi} \int_{0}^{2 \pi} f^{* *}(t) d t, \quad f \in L \log ^{+} L .
$$

Hence to obtain the desired inequality (5) we must show that

$$
\frac{2 \alpha}{\pi}=\mathbf{K}=\frac{1^{-2}-3^{-2}+5^{-2}-7^{-2}+\cdots}{1^{-2}+3^{-2}+5^{-2}+7^{-2}+\cdots} .
$$

Since the denominator sums explicitly to $\pi^{2} / 8$ it will therefore suffice to show that $\alpha=(4 / \pi)\left(1^{-2}-3^{-2}+5^{-2}-7^{-2}+\cdots\right)$.

But from (6), a change of variable and an integration by parts

$$
\begin{aligned}
\alpha & \equiv \frac{1}{2 \pi} \int_{0}^{2 \pi} \log \cot (t / 8) d t \\
& =\int_{0}^{1} \log \cot (\pi s / 4) d s=\frac{4}{\pi} \int_{0}^{1} \frac{\log (1 / u)}{1+u^{2}} d u \\
& =\frac{4}{\pi} \int_{0}^{1} \frac{\arctan u}{u} d u=\frac{4}{\pi} \int_{0}^{1}\left(\sum_{n=0}^{\infty} \frac{(-1)^{n} u^{2 n+1}}{2 n+1}\right) \frac{d u}{u},
\end{aligned}
$$

which integrates term-by-term to the desired value.

To see that the constant $\mathbf{K}$ in (5) is best-possible we make use of the function $f$ constructed in [7, p. 197] as follows. Thus we take $f=2 \chi-1$, where $\chi$ denotes the characteristic function of the interval $[0, \pi]$. Then $|f|$, hence $f^{* *}$, is identically equal to 1 so $\int_{0}^{2 \pi} f^{* *}(t) d t=2 \pi$. But $\tilde{f}=(2 \chi-1)^{\tilde{\chi}}=2 \tilde{\chi}$ whose norm is given by 


$$
\int_{0}^{2 \pi}|\tilde{f}(x)| d x=\frac{2}{\pi} \int_{0}^{2 \pi} \log \cot (t / 8) d t
$$

$[7$, p. 198]. As a result we see from (6) that

$$
\int_{0}^{2 \pi}|\tilde{f}(x)| d x=\frac{2}{\pi}(2 \pi \alpha)=\left(\frac{2 \alpha}{\pi}\right) 2 \pi=\mathbf{K} \int_{0}^{2 \pi} f^{* *}(t) d t
$$

This completes the proof.

The "dual" of Theorem 4 is the following result about conjugate functions of bounded functions.

THEOREM 5. If $f$ is (essentially) bounded on $(0,2 \pi)$, then its conjugate function $\tilde{f}$ satisfies the inequality

$$
\sup _{0<t<2 \pi} \frac{\tilde{f}^{* *}(t)}{1+\log (2 \pi / t)} \leqslant \mathbf{K} \text { ess } \sup _{0<x<2 \pi}|f(x)|,
$$

and the constant $\mathbf{K}$ is best-possible.

Proof. We shall use the identity

$$
\begin{gathered}
\sup \left\{\left|\int_{0}^{2 \pi} F(x) g(x) d x\right|: \int_{0}^{2 \pi} g^{* *}(t) d t \leqslant 1\right\} \\
=\sup _{0<t<2 \pi} \frac{F^{* *}(t)}{1+\log (2 \pi / t)},
\end{gathered}
$$

which is a particular case of a more general theorem of Lorentz [6, Theorem $6]$. The essential content of $(10)$ is that the functional on the right is the norm of the dual space of $L \log ^{+} L$ when the latter space is equipped with the Hardy-Littlewood norm (4).

Let $g \in L \log ^{+} L$ with $\int_{0}^{2 \pi} g^{* *}(t) d t \leqslant 1$. By Theorem 4 its conjugate function $\tilde{g}$ satisfies $\int_{0}^{2 \pi}|\tilde{g}(x)| d x \leqslant \mathbf{K}$. Thus, for the given $f \in L^{\infty}$ we have

$$
\left|\int_{0}^{2 \pi} \tilde{f}(x) g(x) d x\right|=\left|\int_{0}^{2 \pi} f(x) \tilde{g}(x) d x\right| \leqslant \mathbf{K} \text { ess } \sup _{0<x<2 \pi}|f(x)| .
$$

Taking the supremum with respect to all such $g$, and applying (10) (with $F=\tilde{f}$ ) we obtain the desired estimate (9).

The extremal function for (5), constructed in the proof of Theorem 4, is easily shown to be extremal also for (9) (take $t=2 \pi$ ). Hence $\mathbf{K}$ is bestpossible, and the proof is complete.

REMARKs. (i) Burkholder [4] has raised the intriguing question of finding a nonprobabilistic proof of Davis' inequality. We do not know whether it can be derived from our results. However, it is easy to see from (5) that the best constant in (1) is at least $\mathbf{K}^{-1}$ (there is also a direct proof of this, cf. [4]). Indeed, if $f=2 \chi-1$ is the function considered in the proof of Theorem 4, then $\tilde{f}^{2}=-f$, and

$$
\sup _{0<y<2 \pi} y m\{|f|>y\}=2 \pi=\int_{0}^{2 \pi} f^{* *}(t) d t .
$$


Thus, if Kolmogorov's inequality holds with constant $A$, we use that estimate together with (5) to find

$$
2 \pi=\sup _{y} m\left\{\left|\tilde{f}^{\tilde{1}}\right|>y\right\} \leqslant A \cdot \int_{0}^{2 \pi}|\tilde{f}(x)| d x \leqslant A \mathbf{K} \int_{0}^{2 \pi} f^{* *}(t) d t=2 \pi A \mathbf{K} .
$$

Hence $A \geqslant \mathbf{K}^{-1}$.

(ii) From Theorem 3 and the argument used in [1, Proof of Theorem 4.1] it can be shown that in Theorem 2 any constant $A>2 / \pi$ is admissible. That $A=2 / \pi$ is not admissible requires the construction of a counterexample as in [8].

(iii) It would be interesting to know the best constants in conjugate function inequalities between the more general Lorentz spaces or Orlicz spaces. For instance, what is the best constant on the Lorentz space $L^{p q}$ (the $L^{p}$-case is treated in [8]), or in Zygmund's inequality when the space $L \log ^{+} L$ is equipped with the Orlicz norm or the Luxemburg norm? Little seems to be known either about the analogous questions for the maximal conjugate function.

(iv) Finally, we wish to thank the referee for some valuable suggestions regarding the format of the paper.

AdDED IN PROOF. Professor Albert Baernstein II (Washington Univ., St. Louis) has shown me a simple, nonprobabilistic proof of Davis' inequality that depends on the theory of subharmonic functions.

\section{REFERENCES}

1. C. Bennett, Intermediate spaces and the class $L \log ^{+} L$, Ark. Mat. 11 (1973), 215-228.

2. _- Estimates for weak-type operators, Bull. Amer. Math. Soc. 79 (1973), 933-935. MR 47 \#264.

3. - Banach function spaces and interpolation methods. III. Hausdorff-Young estimates, J. Approximation Theory 13 (1975), 267-275.

4. D. Burkholder, Harmonic analysis and probability (preprint).

5. B. Davis, On the weak-type $(1,1)$ inequality for conjugate functions, Proc. Amer. Math. Soc. 44 (1974), 307-311.

6. G. G. Lorentz, On the theory of spaces $\Lambda$, Pacific J. Math. 1 (1951), 411-429. MR 13, 470.

7. R. O'Neil and G. Weiss, The Hilbert transform and rearrangement of functions, Studia Math. 23 (1963), 189-198. MR 28 \#3298.

8. S. K. Pichorides, On the best values of the constants in the theorems of M. Riesz, Zygmund and Kolmogorov, Studia Math. 44 (1972), 165-179. MR 47 \#702.

9. E. M. Stein and G. Weiss, An extension of the theorem of Marcinkiewicz and some of its applications, J. Math. Mech. 8 (1959), 263-284. MR 21 \#5888.

10. A. Zygmund, Trigonometric series. Vol. I, 2nd rev. ed., Cambridge Univ. Press, New York, 1959. MR 21 \#6498.

Department of Mathematics, California Institute of Technology, Pasadena, CaliforNIA 91125 